\title{
Simulation of a microgrid for a non-interconnected zone that integrates renewable energies
}

\author{
German Reina, Mauricio Mauledoux, Oscar A. Avilés \\ Mechatronics Engineering Program, Universidad Militar Nueva Granada, Colombia
}

\begin{tabular}{|c|c|}
\hline Article Info & ABSTRACT \\
\hline Article history: & This paper develops a simulation of a small electrical network (Microgrid) \\
\hline Received Mar 3, 2020 & $\begin{array}{l}\text { that integrates renewable energies, the model of the micro network is made } \\
\text { up of a solar energy source, a wind energy source, an energy storage }\end{array}$ \\
\hline Revised Jul 3, 2020 & element, a non-renewable source such as a diesel generator. The model of \\
\hline Accepted Aug 5, 2020 & $\begin{array}{l}\text { the microgrid represent a non-interconnected area from the electrical network } \\
\text { in Colombia. The non-interconnected areas sometimes depend on unreliable }\end{array}$ \\
\hline Keywords: & $\begin{array}{l}\text { connections to the grid integration of renewable energies could be the best } \\
\text { option to guarantee energy in these sectors and allow generating projects }\end{array}$ \\
\hline Energy & with social impact. A possible solution to this deficit of energy is to \\
\hline Non-interconnected & $\begin{array}{l}\text { supplement the production of energy with renewable energy plants from } \\
\text { resources as sun or wind. The simulated model allowed to study the effects }\end{array}$ \\
\hline Renewable & of the network in island mode and in interconnected mode, showing \\
\hline Smart grid & $\begin{array}{l}\text { the imbalances that can be obtained by integrating renewable energies and } \\
\text { storage systems. It is verified that with an inclusion of more than } 30 \% \text { of } \\
\text { power in renewable energies there is the possibility of having load } \\
\text { imbalances, which affect the frequency and cause instability in the network. } \\
\text { It also verifies how a control system can regulate the load balance but must } \\
\text { interact with the other energy sources. }\end{array}$ \\
\hline
\end{tabular}

This is an open access article under the CC BY-SA license.

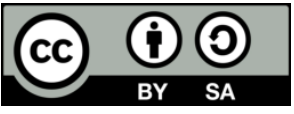

\section{Corresponding Author:}

Oscar A. Avilés,

Mechatronics Engineering,

Universidad Militar Nueva Granada,

Cr 11 No 101 - 80, Bogotá, Colombia.

Email: oscar.aviles@unimilitar.edu.co

\section{INTRODUCTION}

This document develops a model for simulate a microgrid that represent a non-interconnected area from the electrical network in Colombia. It was made to explore the effects that renewable energies have on the electrical network as a power supply with residential loads. It explores the behavior of this energies and verified if the control systems can maintain the balance of load. The simulation is done in two different modes as interconnected or non-interconnected electrical network.

Colombia has a good potential to produce electricity with renewable energies, it does not have seasons and also this country has a location with high annual averages of wind speeds and irradiance from the sun. However, it has difficulties integrating remote regions into the national electricity grid. It non-interconnected zones lack the possibility of energy 24 hours a day. A possible solution to this energy deficit is to supplement the production of energy with renewable energy plants from resources as sun or wind. 


\section{WHY IS IMPORTANT THIS SIMULATION}

\subsection{Non- Interconnected Zone in Colombia}

The government define the term non-interconnected zones (ZNI) in Colombia as following: "For all purposes related to the provision of the public electric power service, ZNI is understood to be municipalities, townships, towns and hamlets not interconnected to the national electric system (SIN-sistema interconectado nacional)" [1]. It ZNI can be defined as microgrid with non-renewable generation sources that can be improved with the inclusion of renewable energies (unconventional) and take advantage of everything that is developed for smart grids and apply the technology in island mode to optimize the fuel from diesel plants that is commonly used as an energy source of energy in these zones. The main characteristics of the non-interconnected zones in Colombia are: areas with unmet basic needs $>77 \%$, are scattered areas (Low Population Density), with a low average consumption level. They have a low payment capacity and a very low level of collection. It has high costs of providing electricity service. High level of losses. Low level of micro measurement. This makes them a perfect scenario for the efficient use of available natural resources and the use of renewable energies.

The non-interconnected areas sometimes depend on unreliable connections to the grid such as the Puerto Carreño in the Vichada zone that depends of a very weak medium voltage circuit from Venezuela. This problem is very important for the development of pilot tests where the application of renewable energies is the best option to guarantee energy in these sectors and allow generating projects with social impact right now. According to the Institute of Planning and Promotion of Energy Solutions for Non-Interconnected Zones IPSE, the non-interconnected areas in Colombia are in 32 Departments. Where there is currently coverage over 17 departments, which is equivalent to $52 \%$ of the national territory is included 90 Municipalities, 20 Special Biodiversity and Border Territories, 1,448 localities ZNI, 39 Municipal Headquarters: 5 departmental capitals [1].

\subsection{Why a non-interconnected zone is a type of microgrid?}

A microgrid is a section of an electrical network that can be connected to a main network and operate autonomously, feeding its own loads from its own sources of energy for some periods of time. When a microgrid is operating autonomously without connection to the main network, it is said to be operating in island mode ("islanded"). The microgrid is controlled by a real-time monitoring system that concentrates on regulating the voltage and frequency, this is achieved by controlling the different sources of energy and always seeking to balance the load and energy generated, as well as the energy injected from the main network. This controller should generally consider the load profiles, define an operating cost for each of the sources, estimate the amount of solar energy and wind energy. All this application only can be possible If the different resources or multi agents in the network send and receive information to all the others. The interoperability is being useful to maintain the balance of load in the electrical network.

A microgrid can decide to isolate itself from the main network (grid) when there are faults in the main network or when the quality of the energy is very poor, with this strategy it is sought that the users can have a better reliability and quality in the energy that is associated to the loads of the microgrid. This capacity is also part of the skills that allow to this type of networks, to integrate sources with renewable energy, to take electrical power from the main network (grid) while there is no capacity to be self-sufficient. Microgrid generally have backup sources such as diesel generators, gas turbines. Their location is in remote places where the loads must be fed with greater reliability than the confidence that the network can provide. It generally applies to hospitals, military installations and some manufacturing processes [2].

Microgrid are constantly in research and development and there are several pilots that are being developed [3-6], leaving an operating cost of around 5 USD per Watt of capacity. These micro networks and research projects are focused on high reliability for the loads they handle, such as hospitals or military bases, but due to their high cost they have not been taken to other users. The simulation model is designed to represent the electric grid of the non-interconnected area of Puerto Carreño in Colombia as a Microgrid that integrate renewable energies trying to explore the effects and understood the problems to improve the performance of this networks.

\section{CHALLENGES IN THE INTEGRATION OF RENEWABLE ENERGIES}

The main effect of the inclusion of renewable energies in the network is that they are basically more prone to instability or variations in their quality parameters, it microgrid need to react quickly with control actions on their own maneuvering equipment and eventually involve all those that are interconnected. The first challenge of the electric networks that integrate renewable energies, is to regulate the power that is delivered to the end user at the moments when there is no availability of alternative sources (for example: 
in the absence of sunlight or absence of wind). This means that the planning of the required energy must consider the seasons of the year, and probabilistic studies of the wind projections that are available or the solar radiation that is expected at a certain point. Some renewable energies depend on natural phenomena, the available energy is conditioned to the available resource at a given moment [7-11]. An electrical network based only on renewable energies such as wind energy, solar radiation will have problems to feed the load in a constant manner as shown in Figure 1, production and consumption varies over time.

Elements that allow the regulating of energy delivered to the end user must be integrated in the microgrid, because in case of absence of solar radiation [12] or wind [13] these systems are unusable to generate energy and the energy received by the end user will be zero [14]. The variability of the network and the fast reacting that now is required for maintain the balances between the load and the renewable energy sources is the principal problem to be simulated. In some remote places there are microgrid composed only of renewable energy that must provide energy to the user 24 hours a day, these must necessarily have devices that allow to the energy generated to be stored or be supplemented with other sources of generation [15] for its proper functioning.

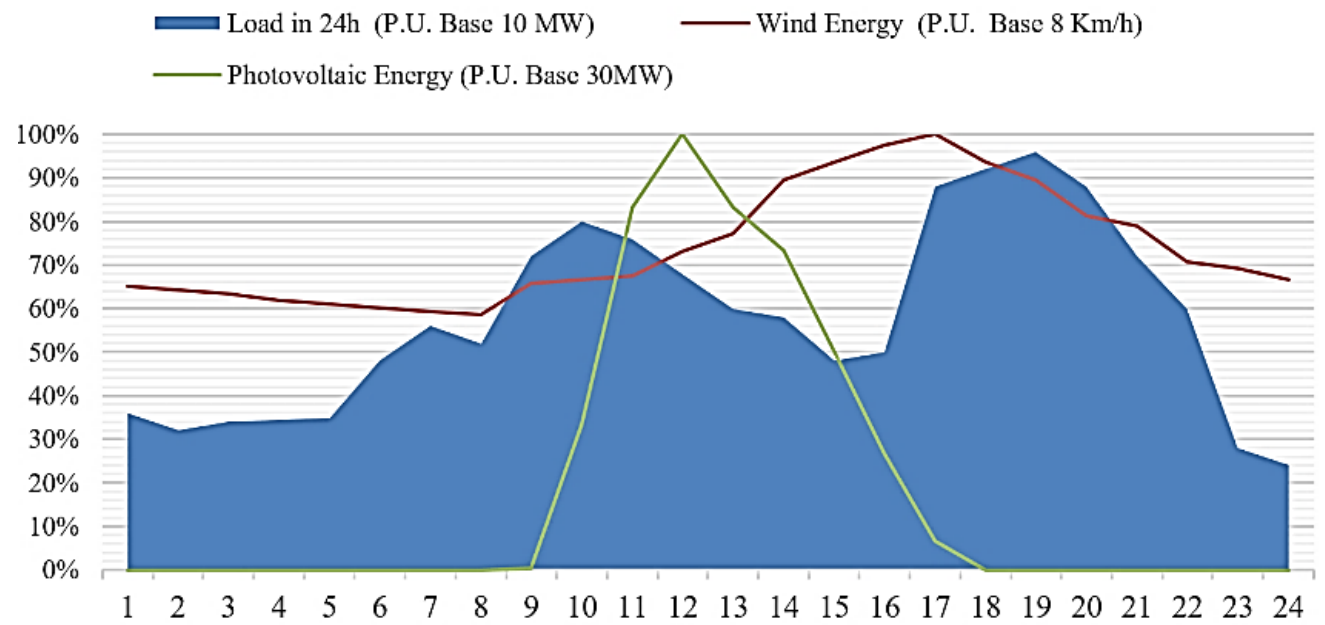

Figure 1. Typical energy production and consumption in a Day $(24 \mathrm{H})$

The solution for integrating renewable energies and regulating fluctuations is called smart grids. This is a type of network that can coordinate the available energy sources and restore the stability in the network. Smart grid will help to increase the level of penetration of renewable energies, IRENA (International Renewable Energy Agency) [16] declare that with levels of penetration higher than 30\%, this information exchange technology is essential for the proper functioning of electrical grids.

\subsection{Smart grid as solution}

The main characteristic of a smart grid is to use technology to acquire information instantaneously to achieve the balance between generation and demand, in this way it helps to make decisions based on the information received, and keeps the system operating with the highest efficiency. The technology can be applied in generators and consumers. The desired performance of a smart grid are: The generation of energy is already distributed and consumers can contribute energy to the network; the flow of energy is in two directions from generators to consumers, and consumers to generators; Consumption control is based on real-time and historical data; however, the network can have rapid variations in power flow; It must maintain the stability of the network, even when the available sources are variable and depend on natural elements; Depending on the response times required, energy must be stored in some form; The generated voltage, frequency and topology of the network are monitored.

This supervision is semi-automatic or fully automatic and should have more advanced control. Even automatic topology changes are allowed; The consumer is an active entity and can generate energy for the electricity network. The equipment that integrate a smart grid must be has: 1) the ability to communicate between the different components and agents; 2) data management in real time; 3) Collection of information for taking control actions; 4) Execution of control actions. 


\section{THE MODEL OF THE MICROGRID}

\subsection{Electrical connection like a non-interconnected zone}

The simulation model is designed to represent the electric grid of the non-interconnected area of Puerto Carreño in Colombia. The Figure 2 [17, 18] shows the single line diagram, this electrical grid has the following characteristics: The microgrid has a connection from an electrical network at $34.5 \mathrm{kV}$, this energy is sent to a distribution substation at $13.2 \mathrm{kV}$ (Grid Connection), the Diesel power plants in $480 \mathrm{~V}$ are connected to the grid through a step-up transformer to the $13.2 \mathrm{kV}$ medium voltage busbar, the loads are connected by a pole-mounted reducer transformer that reduces the voltage from $13.2 \mathrm{kV}$ to 208/120 V to be used by the residential load.

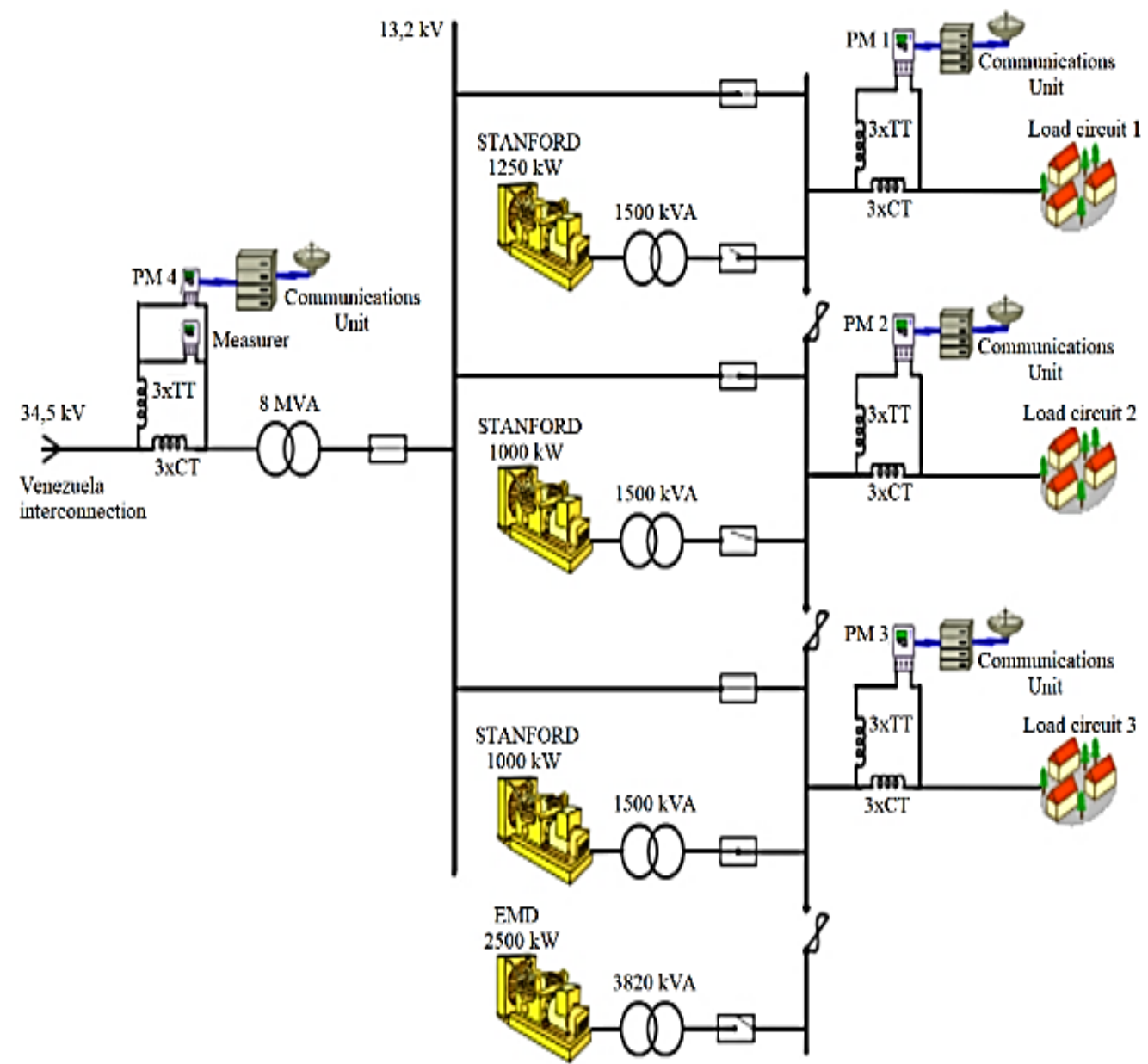

Figure 2. Puerto Carreño grid, single line diagram

For the electrical network, the simulation emulates the connection of the power system of Puerto Carreño, where the loads are approximately 5 MVA, the network arrives from Venezuela through a medium voltage line. The generators are connected to the network through an elevator transformer. The nominal rates and capacities are proposals in the model showed in the Table 1. In the upper part of the table the source is indicated. The first row is the nominal phase-phase voltage that will be used for the electrical connection, in the lower part is show the nominal power of each source or load.

Table 1. Voltages and Powers of the sources that make up the micro network.

\begin{tabular}{cccccc}
\hline & Diesel generator & Wind farm & Photovoltaic Park & Storage & Load \\
\hline Nominal Voltage [Vn] & $480 \mathrm{~V}$ & $13.8 \mathrm{k} \mathrm{V}$ & $13.8 \mathrm{kV}$ & $208 / 120 \mathrm{VAC}$ & $208 / 120 \mathrm{VAC}$ \\
Nominal Power [Sn] & $10 \mathrm{MVA}$ & $2 \mathrm{MVA}$ & $2 \mathrm{MVA}$ & 400kVA & 5MVA \\
\hline
\end{tabular}


The size of the load is define by the report from IPSE in the monthly telemetry report of the non-interconnected area of Puerto Carreño 2014 [17, 18]. The power of the Diesel generator is the sum of the plants that this network already has. The elevating transformers are installed to include the renewable sources at a typical distribution voltage used in Colombia assuming that they are far from the consumption centers, as well as implements the typical reducing transformers that exist in the distribution networks close to the load. The microgrid has the capacity to connect to the network.

The electrical connection consists of three nodes (BUS_1, BUS_2, BUS_3) shown in Figure 3, the first is the diesel generation node at $480 \mathrm{~V} \mathrm{AC}$, this point has a small load that represents the auxiliary services of the machine and from where the parameters for frequency regulation will be taken. The distribution substation is represented as the BUS_2, where the diesel generator is connected through the $20 \mathrm{MVA} \mathrm{Yg/} \mathrm{Yg} \mathrm{elevator} \mathrm{transformer.} \mathrm{In} \mathrm{this} \mathrm{busbar} \mathrm{the} \mathrm{sources} \mathrm{of} \mathrm{the} \mathrm{electric} \mathrm{network,}$ the wind farm plant and the photovoltaic farm are connected. The busbar at $13.8 \mathrm{kV}$ and has a reference to ground through a ZIG-ZAG transformer of $10 \mathrm{MVA}$, this is installed in case the diesel source was isolated and the system is floating. This bus feeds the load node through a reducing transformer from $13.8 \mathrm{kV}$ to $208 \mathrm{~V}$ with a capacity of $20 \mathrm{MVA}$ Yg / Yg. The BUS_3 represents the lines of distribution up to the loads. The loads are connected to 208/120 VAC as well as the 400-kVA distributed storage system [19].

An important point in this design is that it took into consideration the high cost of large distributed storage systems, which at this time reduces the storage capacity of this technology, since they will not be implemented in the current solutions until their price is more affordable, this would give an approximation to the real environment that may be in the near future In order to simulate the effect of renewable energies a level of penetration of renewable energy sources exceeding $30 \%$ was proposed, this is represented as follows, 2 MVA of the wind farm plus 2 MVA in the solar plant for a total of $4 \mathrm{MVA}$, which is equivalent to approximately $40 \%$; the non-renewable generation source is $10 \mathrm{MVA}$; The storage capacity is $400 \mathrm{kVA}$ (Energy Storage 4\%) and finally the consumption of the load is 5 MVA.

\subsection{Simulation considerations}

For the simulation of this microgrid, the Matlab program was used, especially the Simulink tool and its Simscape ${ }^{\text {TM }}$ Power Systems tool block. The electrical network was modeled on the examples of this library and the tool blocks that this program offers. The simulations are done with phasors for better times of simulation and because this representation facilitates the implementation and calculation of the three-phase power systems. The network of the project was adapted and presented in Figure 3, the electric model of the network was proposed and interconnected to run a load flow, this allows to simulate different scenarios, it was decided to use a time rage of 24 hours where the load flow is run on three nodes Bus_1 Diesel plant, Bus 2 renewable sources and Bus 3 load and storage systems. The model of the Matlab example library with simulation for 24 hours, was adapted to correspond to the network designed for this specific microgrid.

The simulation of the exchange of information is between the storage system and the diesel plant, since the grid network is modeled as an infinite source and over which there is no control. Wind power plants and solar energy behave as independent systems or disturbances where it is not possible to anticipate the energy they will produce. The control strategy consists of keeping the frequency and voltage on BUS_3 as close as $60 \mathrm{~Hz}$ and at 208 VAC. The simulator does not allow to use an OSI exchange model based on the IEC 61850 standard [20, 21], to replace this concept in the simulation it is assumed that the information has no delays and that it is exchanged directly, that is, there is an ideal process bus for the reception and sent information to each controller. The information was exchanged using global tags, these can be visualized by other components

Due to stability problems in the load flow generated when executing the switch maneuvers (Transient that occur in the opening or closing maneuvers - TRV), there were some difficulties, which caused that the program did not find the solution when simulating the load flow. As a solution to this problem, the maneuvers were disabled, and the switches were removed from the generation sources. Only the switch associated with the ideal source (Grid) is implemented, which will be controlled to make the disconnection in the case of island mode

The simulation models are:

a. Mode Island: When the switch associated with the network is opened and the system is working alone through the generator and renewable sources.

b. Cogeneration: The network is connected, and the controller is modeled to minimize the use of fuel but maintaining stability in the frequency 
The load is of residential type and two different ways of simulating it were implemented, one as a constant system and the other with a variable load profile during daylight hours. Profiles were also generated for the wind and for the sunlight that feed the renewable energies, taking as a base the local information of the installation through the historical data of the IDEAM and its virtual atlas [22, 23].

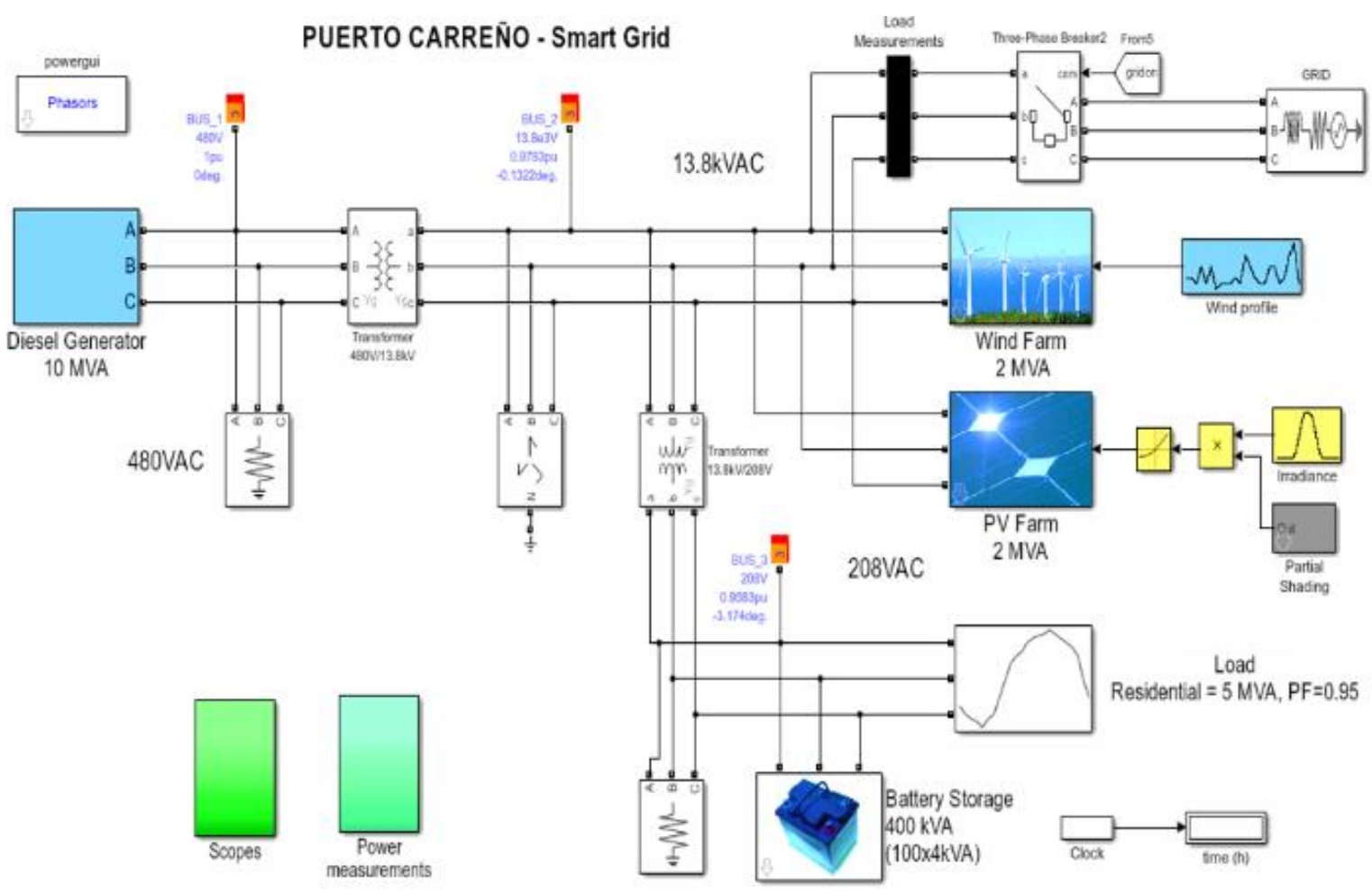

Figure 3. Microgrid simulation diagram

\subsection{Models for simulation}

The choice of generation sources is made by recreating the Colombian scenario, which has several interconnected and non-interconnected areas that can make use of renewable resources and could be implemented in these areas in the future.

\subsubsection{Diesel plant}

The model for the diesel plant is shown in Figure 4(a), it consists of an engine that sends mechanical power to a synchronous machine, the governor is responsible for regulating the power according to a control criterion, in this case is the speed of the rotor (associated with frequency). The parameters of a machine are taken under the norm IEEE recommended practice for excitation system models for power system stability studies, developed by G. Sybille (Hydro-Quebec), Tarik Zabaiou (ETS) - power_machines [24].

The engine is represented inside the governor which generates power that delivers to the synchronous machine, it also has an excitation system which is responsible for controlling the voltage of the machine (associated with the amplitude) and power. This model is taken from the Matlab library and adapted for this simulation. The regulator of the machine has a feedback from the output of the synchronous machine block and calculates the error, to send the desired torque and thus close the control loop.

This model has a phasors meter that later is used to decompose the active power $(P)$, reactive $(Q)$ and apparent $(S)$ provided by the system. The calculation of the power used in the simulation assumes that it is a balanced system, in which by the interposition theorem there is only the positive sequence for which this calculation method is applied. The Figure 4(b) show the calculation system. The power factor (angle) is taken from the difference of the angles of the phasors representing the current and the voltage. 


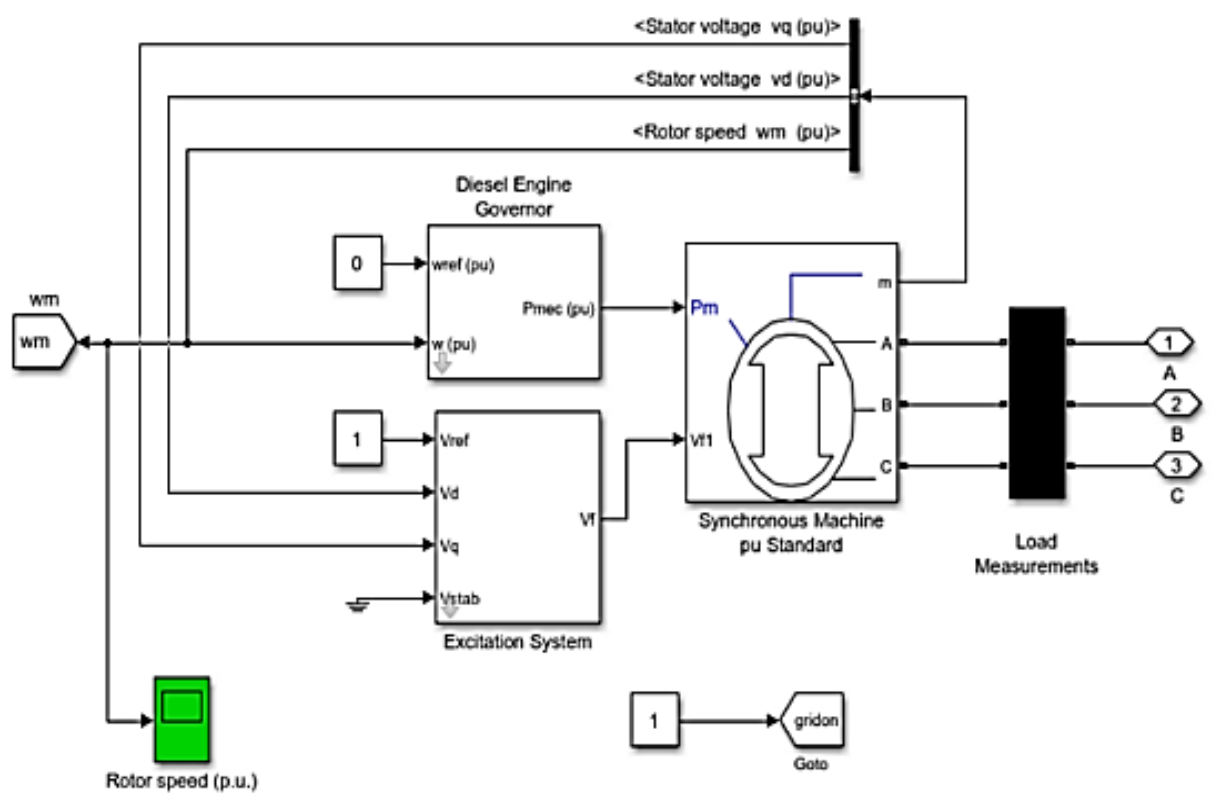

(a)

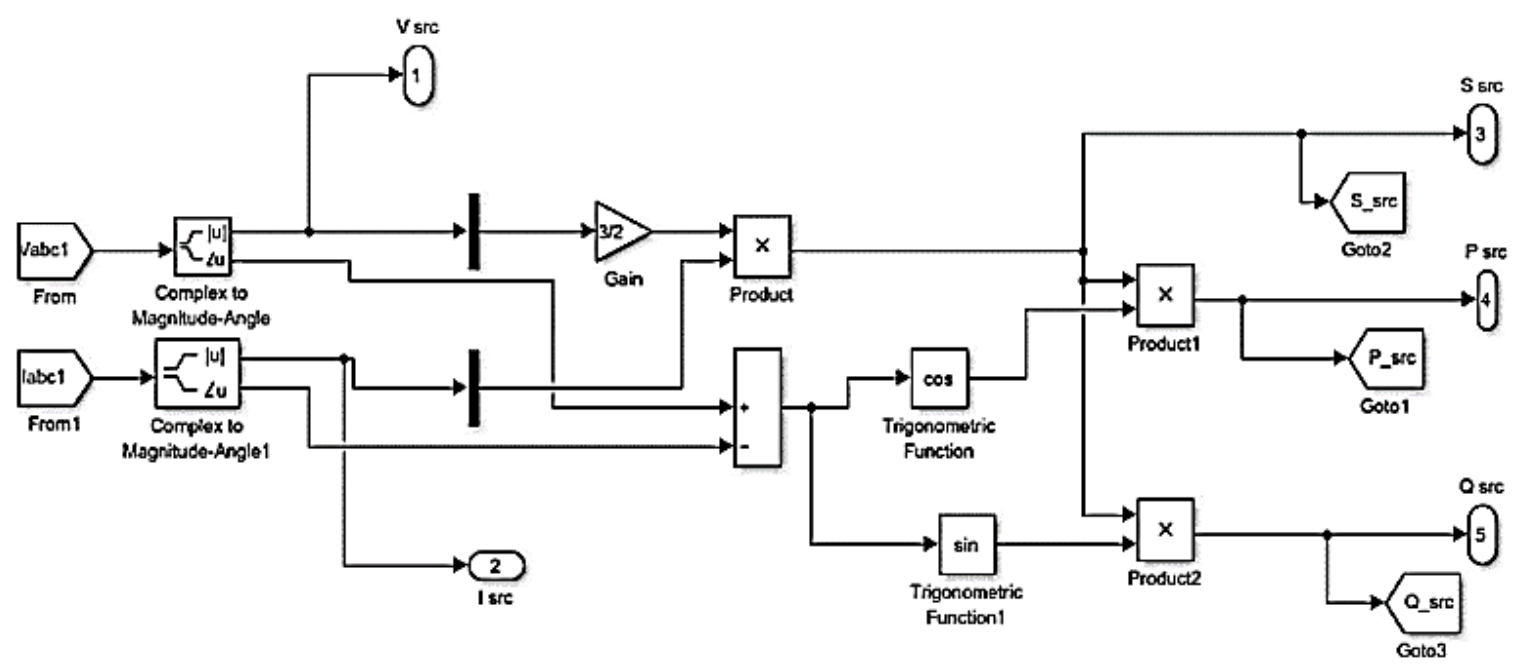

(b)

Figure 4. Models for simulation, (a) Simulation model for the diesel plant implemented in matlab, (b) Calculation of the power based in voltages and currents

\subsubsection{Win plant}

The wind generator is represented as a cubic relation between the wind and the power output with a certain efficiency and a fixed reactive power. For the simulation, a velocity profile was generated based on the averages shown in the IDEAM virtual atlas, where the wind speed increases at nighttime. For the generator, a simplified system was used, which converts the power of the DC system into a three-phase power generator balanced in $\mathrm{AC}$; this modeled inverter through a relationship between the voltages that the system has and the currents that must be injected into the system and the powers in DC, allows the simulation to be faster and generate the three-phase power in the system with only knowing the power in DC generated

In order to better approximate the model of the wind farm, the example proposed by Matlab is implemented, by incorporating a protection over speed of the park, at the moment that the programmed limit is exceeded, the system cuts the power generation, until the wind speed decrease below the adjusted value. In the Figure 5 is showing the model that is used to generate an apparent three-phase power in AC with a desired power in DC [25]. 
The power calculated to the network is converted into three-phase power assuming that the system is balanced, so that only power is generated because it is in zero sequence. Where the threephase voltages $V_{a b}$ and $V_{b c}$ are measured, the positive sequence voltage $V_{1}$ is calculated using the rotation operator " $a$ " (1).

$$
a=-\frac{1}{2}+j \frac{\sqrt{3}}{2}
$$

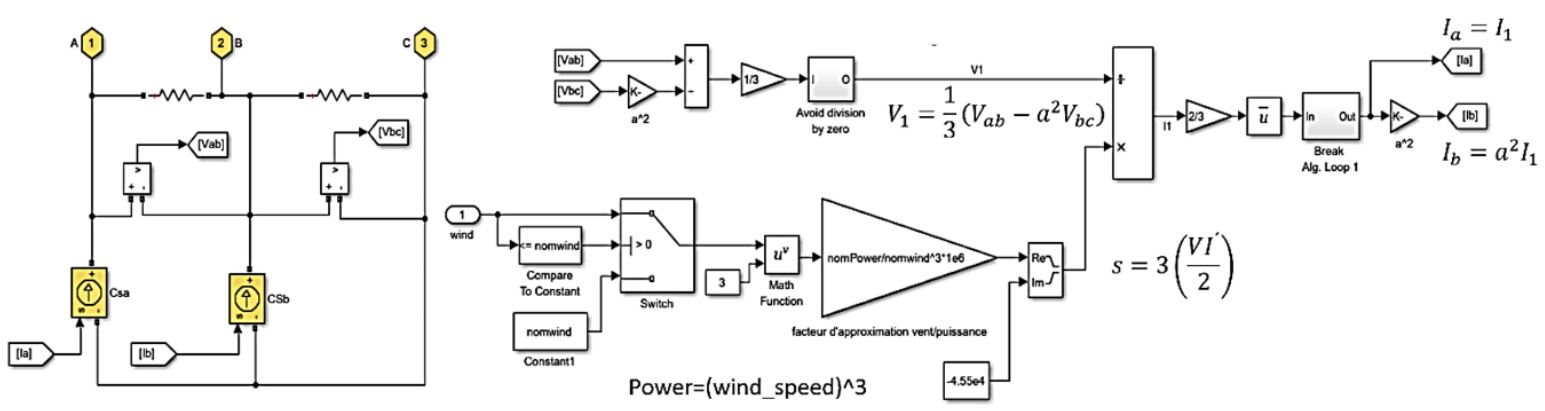

Figure 5. Wind plant model

Once you have the three-phase power $S$ for the balanced system, you proceed to calculate the positive sequence currents $I_{1}$ and $I_{2}$ that will give the value of the three-phase current generated by the source. This concept is used throughout the simulation to represent DC to AC power converters. This model is used to generate an apparent three-phase power in DC with a desired power in DC. After generator sends the power quantity, the system must adapt the voltage to a value that allows it to be connected to the transmission network in $13.8 \mathrm{kV}$ simulating the required substation in this type of plant, it also includes a load that represents the losses and elements consumed by the own systems. The ideal source is placed so that the system can calculate values of $V_{a b}$ and $V_{b c}$ as reference at the beginning of the simulation, see Figure 6.

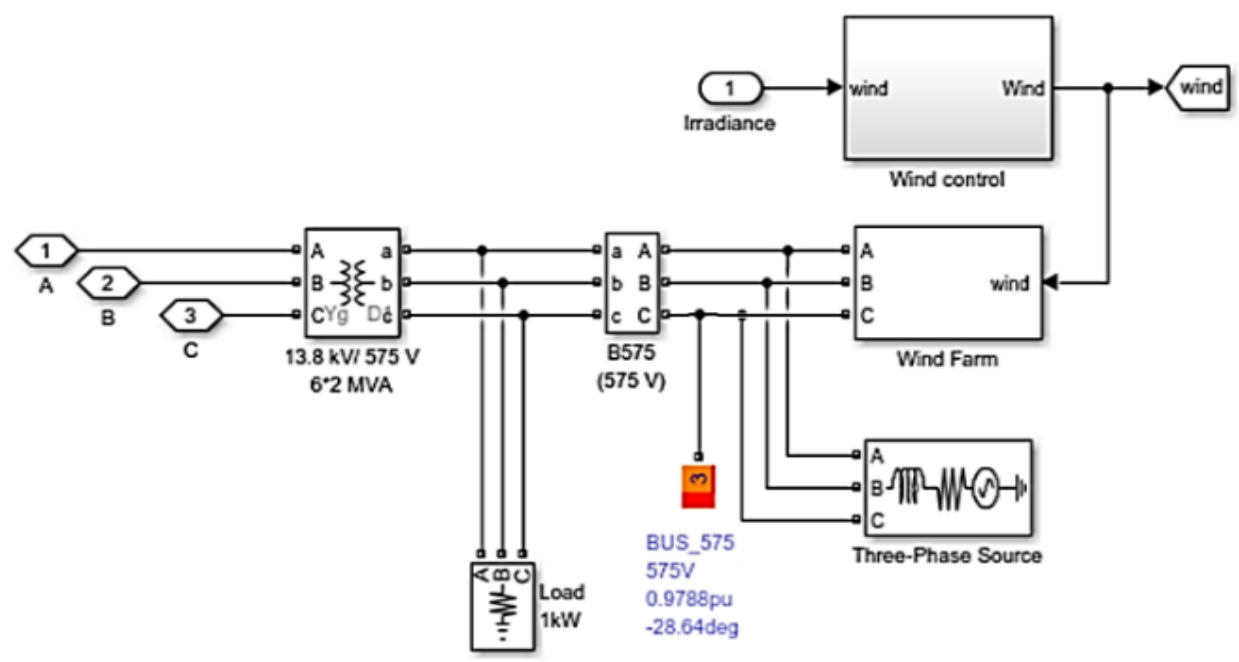

Figure 6. Electrical connection for the wind plant

In this simulation, the reactive generation capacity is limited, as is the case with wind turbines in real life. In summary, the implemented model allows:

a. Simulate the trips by over speed.

b. Deliver three-phase power as a relation to wind speed.

c. Limit the reactive power delivered $(Q)$ 


\subsubsection{Photovoltaic plant}

The model for the solar generation plant is composed of a relation between irradiance and power, where the power is calculated depending on the area to be used and the efficiency of the cells the concept is shown in Figure 7. With this assumption simplifies the model, assuming that the modules and arrays of cells are grouped and deliver a DC voltage with a certain power. The output of the three-phase generator is connected to a load that represents the own services, in addition to the ideal source for the calculation of the voltages $V_{a b}$ and $V_{b c}$ shown in Figure 8. This output at $575 \mathrm{~V}$ is raised to $13.8 \mathrm{kV}$ through the elevator transformer, which connects to the network. In this model, the generation of reagents is also limited. To simulate the electrical behavior of the PV Plant.

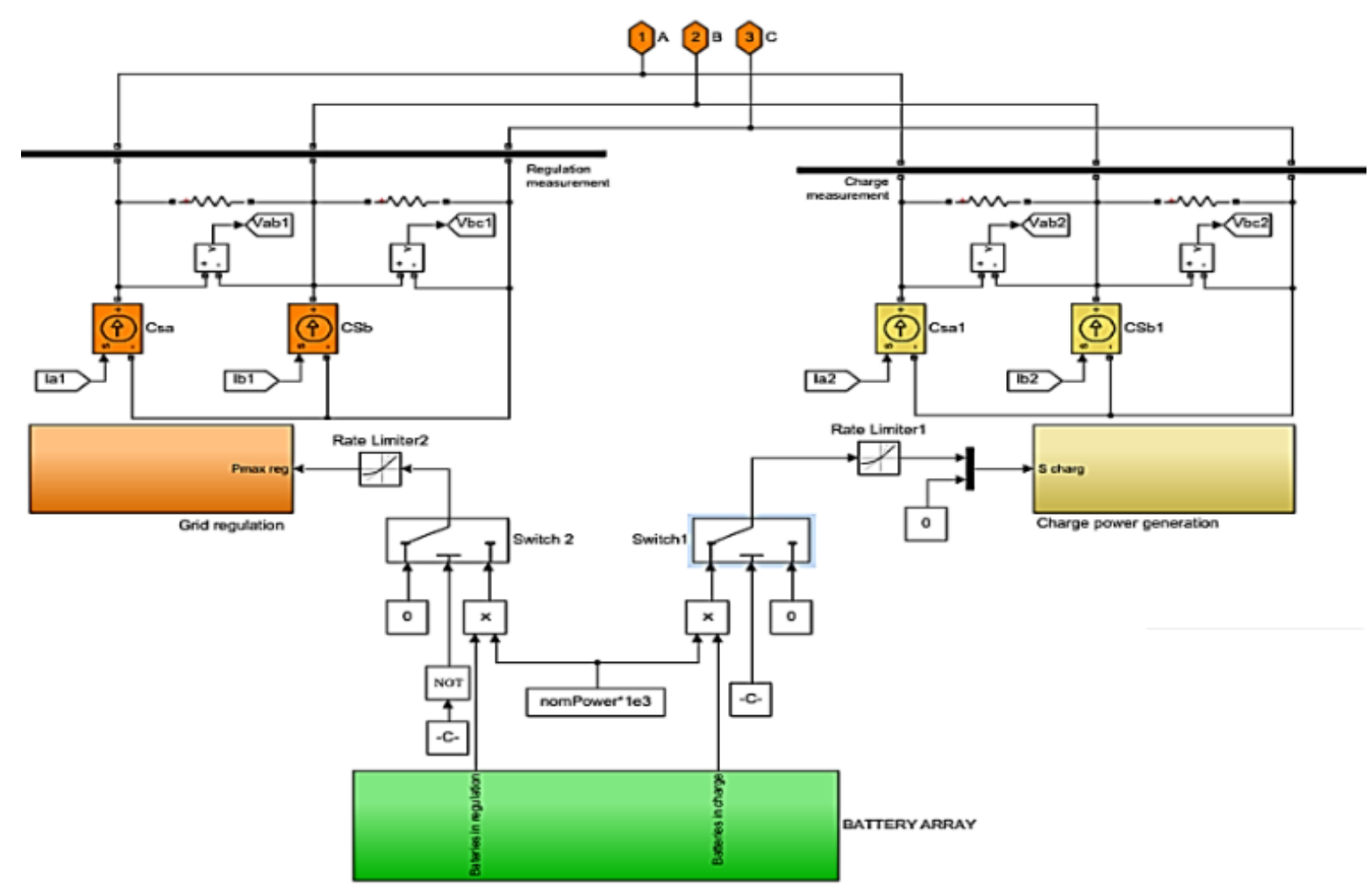

Figure 7. Energy storage model based in a matlab example
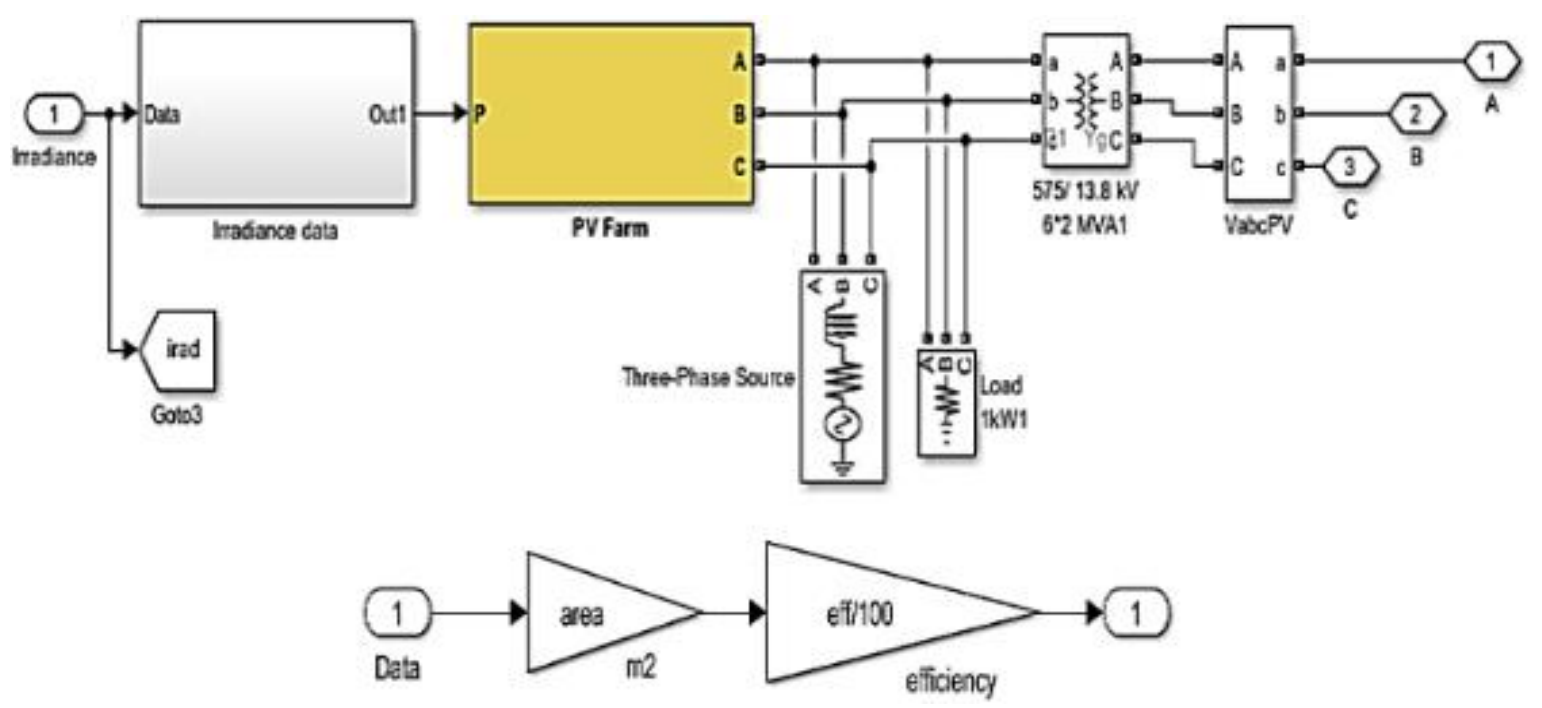

Figure 8. Electrical connection for the PV plant 


\subsubsection{Energy storage}

The energy storage system was represented in a set of battery modules, grouped in blocks to increase the variability, a random function is included to represent in the model that the blocks are in different states of charge, and the blocks can be randomly in charge and regulation mode. The controller takes the blocks that are in regulation mode (SOC> 85) and they have power to contribute to the network, the blocks that are below the limit value, absorb power from the electrical network.

Two different converter blocks are used that represent the system, the first is an AC/DC converter for the chargers and the second is the DC/AC converters for take energy from the batteries to the electrical system. The modules have the possibility of being connected or disconnected from the network through the switch to simulate the connection or disconnection of the storage systems offering the possibility of switching each battery block and disabling the regulation. The Figure 8(b) show the storage model.

\subsubsection{Residential load}

To represent the residential load, two options were taken, the first option allows the simulation of a constant load and the second allows simulating a variable load profile over time. The second option is a residential charge that increases consumption at night and reduces in the early morning hours Figure 9.
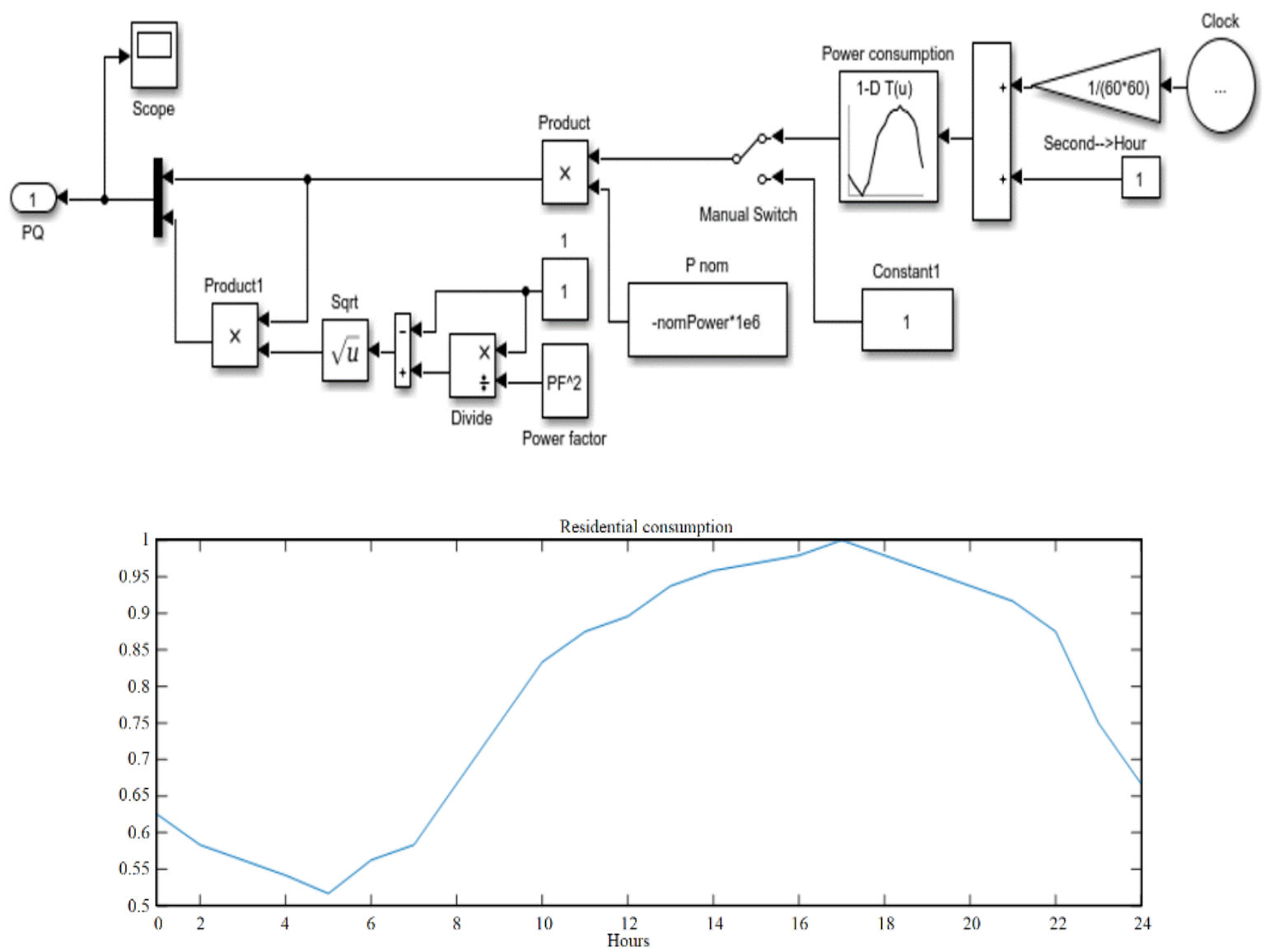

Figure 9. Power consumption for a residential load in 24 hours

\section{SIMULATION}

The microgrid that was implemented for the simulation has two elements that can regulate the amount of energy delivered to the load, the first element is the Diesel plant and the second is the energy storage equipment. The source of the electrical network (GRID), although it delivers energy cannot be regulated, only open or close maneuvers can be carried out controlling with the associated maneuvering equipment. 
The diesel generator controller informs the other systems of the speed of the rotor $\left(\omega_{m}\right)$, so that in this case the controller of the storage system can verify the available accumulated energy and initiate control of the frequency of the network. These two controls work in parallel to keep the network stable. When the system is in cogeneration reducing the consumption of the network, the governor is programmed to maintain the stability of the network with the motor to the minimum $\left(\omega_{m}\right)$ ref $=0$ and only regulating reagents, which makes the generator only work in cooperation with the storage system in cases where the network presents events that may destabilize the micro network, in this case, the plant contributes reagents to the network and complements what is consumed to maintain the stability of the frequency.

In island mode simulation, the microgrid behaves like a weak network; that means, it has small generation sources sensitive to power oscillations, this makes the system very sensitive and exposed to instabilities when loads behave very dynamically. In this case, the plant controller must send the necessary commands to try to maintain the stability of the network. In parallel, the controller of the storage system verifies the behavior of the frequency $\left(\omega_{m}\right)$ and provides energy to keep the system stable. They are basically two controllers working in parallel to keep the frequency within the operating range. Variations in the active power mainly affect the frequency of the system. Variations in reactive power are less sensitive to changes in frequency and locally affect the magnitude of the voltage [6].

The main regulator in this mode is the governor of the machine that tries to maintain the frequency by supplying power to the network. The design of this type of regulators is basically based on PD controllers, the recommended regulator was implemented as IEEE recommended [24]. It is show in Figure 10, the parameters of a machine are taken under the IEEE standard Recommended Practice for Excitation System Models for Power System Stability Studies, developed by G. Sybille (Hydro-Quebec), Tarik Zabaiou (ETS) - power machines. Adjustments were made to avoid overshoots and minor adjustments for the specific load to adjust the parameters to the size of the network and the load [26]. In cogeneration mode the parameters of the governor are modified to try to maintain the stability of the network by reducing the fuel consumption of the machine but keeping the frequency in a stable state. It seeks to maintain the lowest contribution of the machine to the network by taking energy from renewable energies and the electricity grid.

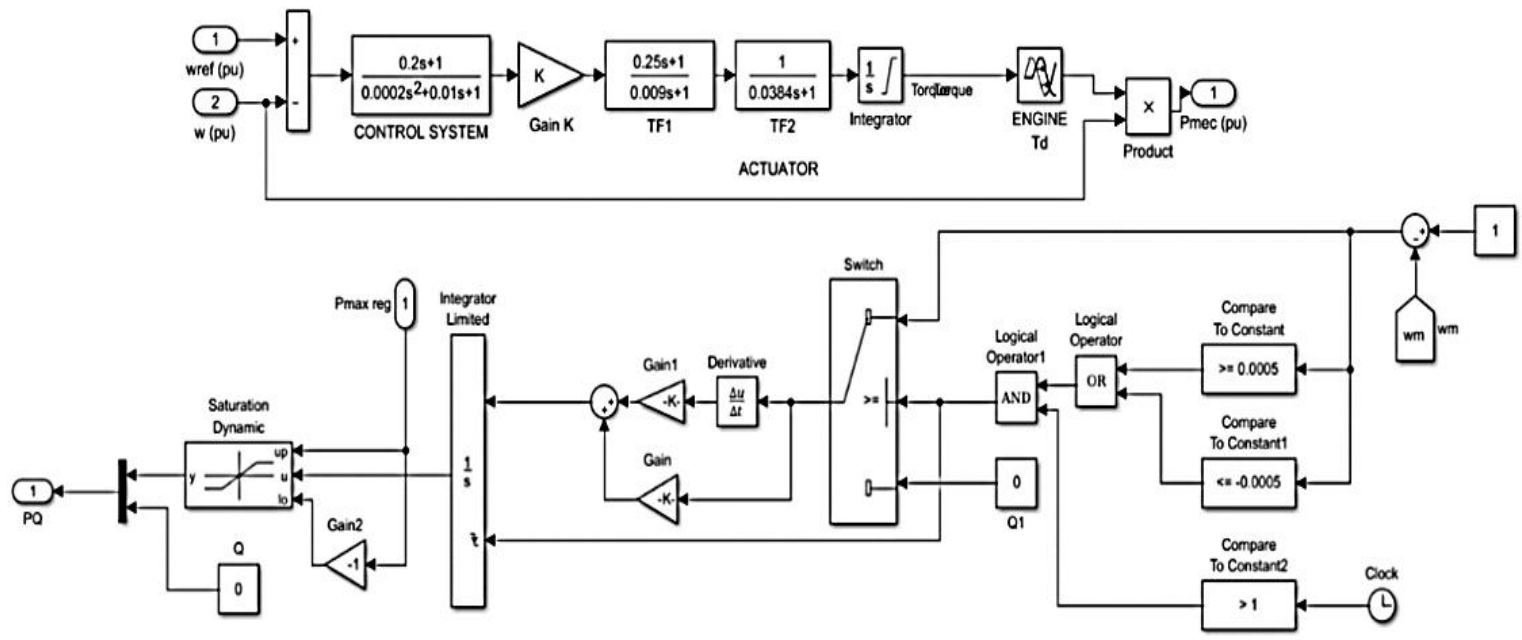

Figure 10. Controls for the microgrid

\subsection{Models for simulation}

This simulation focus on representing the non-interconnected areas of Colombia such as Puerto Carreño, Vichada. Scenarios were executed where the power contributed by the network was modified, the load profile was modified. For others the battery regulation systems were turned off or on. This flexibility gives the opportunity to develop different scenarios of simulation. They are planted as critical scenarios to analyze

a. System disconnected from the network, Island mode with variable load.

b. System disconnected from the network, with regulation by batteries and variable load.

c. System connected to the network, with regulation by batteries and variable load to minimize the power of the plant, decreasing the consumption of the diesel plant.

Simulation of a microgrid for a non-interconnected zone that integrates renewable energies (German Reina) 
The simulation runs in a time interval of $24 \mathrm{~h}$ and with phasor values to optimize the execution time. Approximately 5 minutes per stage. At the beginning of the day, the simulated load is $4 \mathrm{MVA}$, where wind energy has two crests of 1.5 MVA generation. In this period of time, the diesel plant assumes the load of the system [27, 28].

The Figure 11 shows the apparent power ( $\mathrm{S}$ in MVA) of the system for each of the sources in the network. The total apparent power generated is represented in green, the power provided by the Diesel plant in yellow, the wind farm in blue, the photovoltaic park in red. In this simulation, there is no external network power, that is, it is an island mode simulation.

The simulation runs in a time interval of 24 hours and with phasor values to optimize the execution time. Approximately 5 minutes per stage. At the beginning of the day, the simulated load is 4 MVA, where wind energy has two crests of 1.5 MVA generation. In this period of time, the diesel plant assumes the load of the system. The photovoltaic energy only begins to contribute from 8 a.m. in the morning $(2.8 \times 104$ in $x)$ and reaches its peak at noon. There is a cloudiness at 10 a.m., its maximum power is 1 MVA. In the afternoon hours $\left(60 \times 10^{4}\right)$ the load is increased, and the generation reaches its maximum peak of 6 MVA. At 10 o'clock at night a trip of the electric plant is presented due to excessive wind speed, this generates a significant fall and oscillation in the stability of the network.

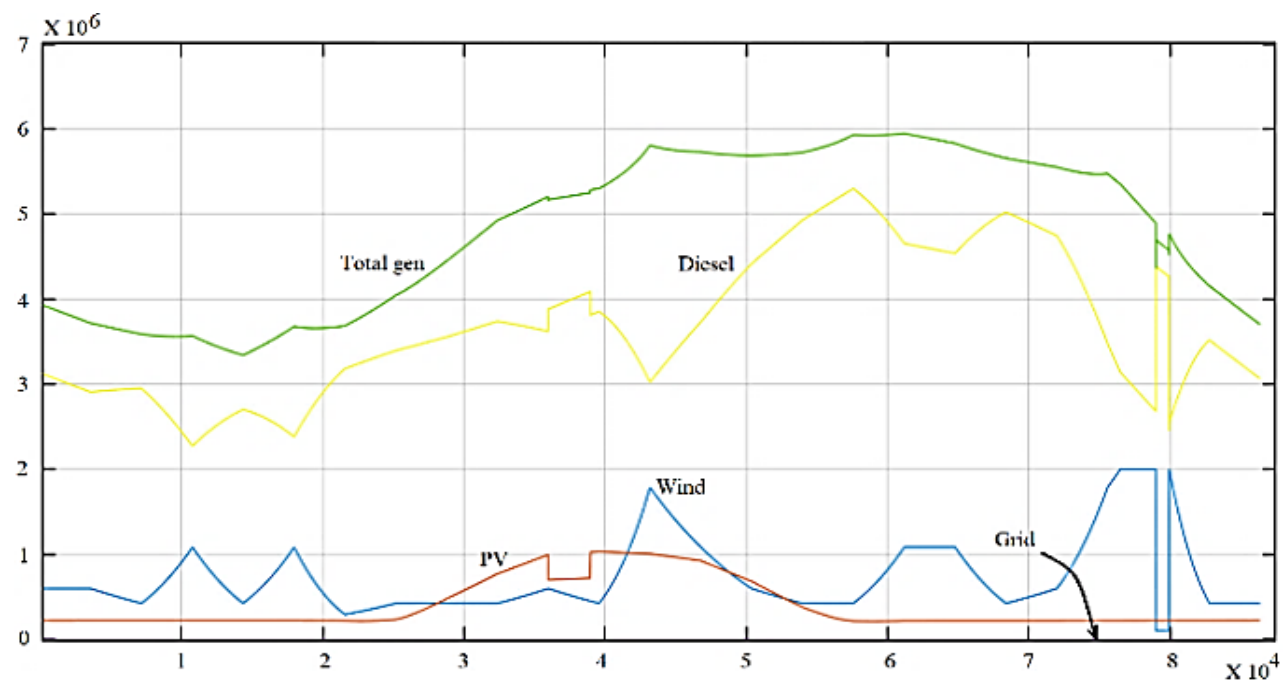

Figure 11. Apparent power simulation result (S) in $24 \mathrm{~h}$

\subsection{Island mode}

Figure 12(a) shows the result of the sum for apparent power (S in MVA) that the system generates, in the center is the graph that represents, the power demanded by the load and in the last box the figure show what is generated what is demanded by the load. The difference between the two curves is given by the loads that represent the losses in the lines or transformation equipment and services of each of the generation sources. The load curve is residential, and its lower limit is 2.8 MVA and greater limit is more than 5.2 MVA.

The controller manages to maintain the load despite the variations in the wind and solar renewable energy plants. Maintains the stability of the system. The biggest disturbance occurs with the 2 MVA shed that is produced by the excess of speed of the wind at 10 p.m. ( 8 x104 in $x$ axis). Some peaks are show for this cause in the sum of power, however, the controller manages to maintain the stability of the network [29]. The generator maintains a production of approximately 4 MVA. The scenery show in the Figure 13 of the simulation does not have the energy storage system since it had as objective to show the stability of the network and the over peaks in the case of load shedding. These over peaks are shown in the Figure 12(b) where their greatest differences with the reference occur, in the moment where increases of instantaneous charges or the power supplies are affected by natural phenomenon that occur with the cloudiness of the photovoltaic cells and with the shutdown of the wind farm by over speed $(3.8 \times 104$ and $8 \times 104$ in $x)$, despite these the stability are maintained between $0.08 \mathrm{~Hz}$. 

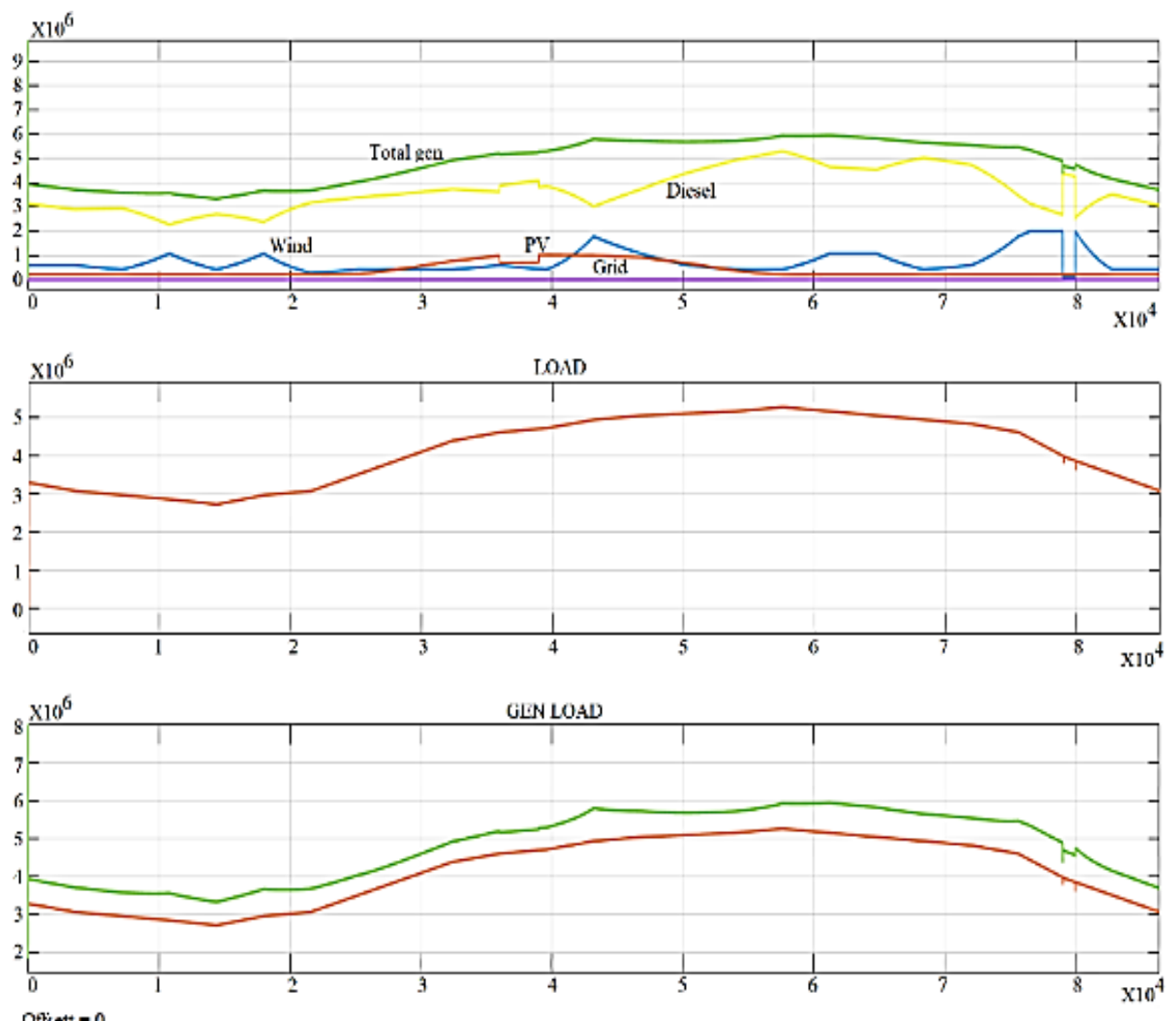

(a)
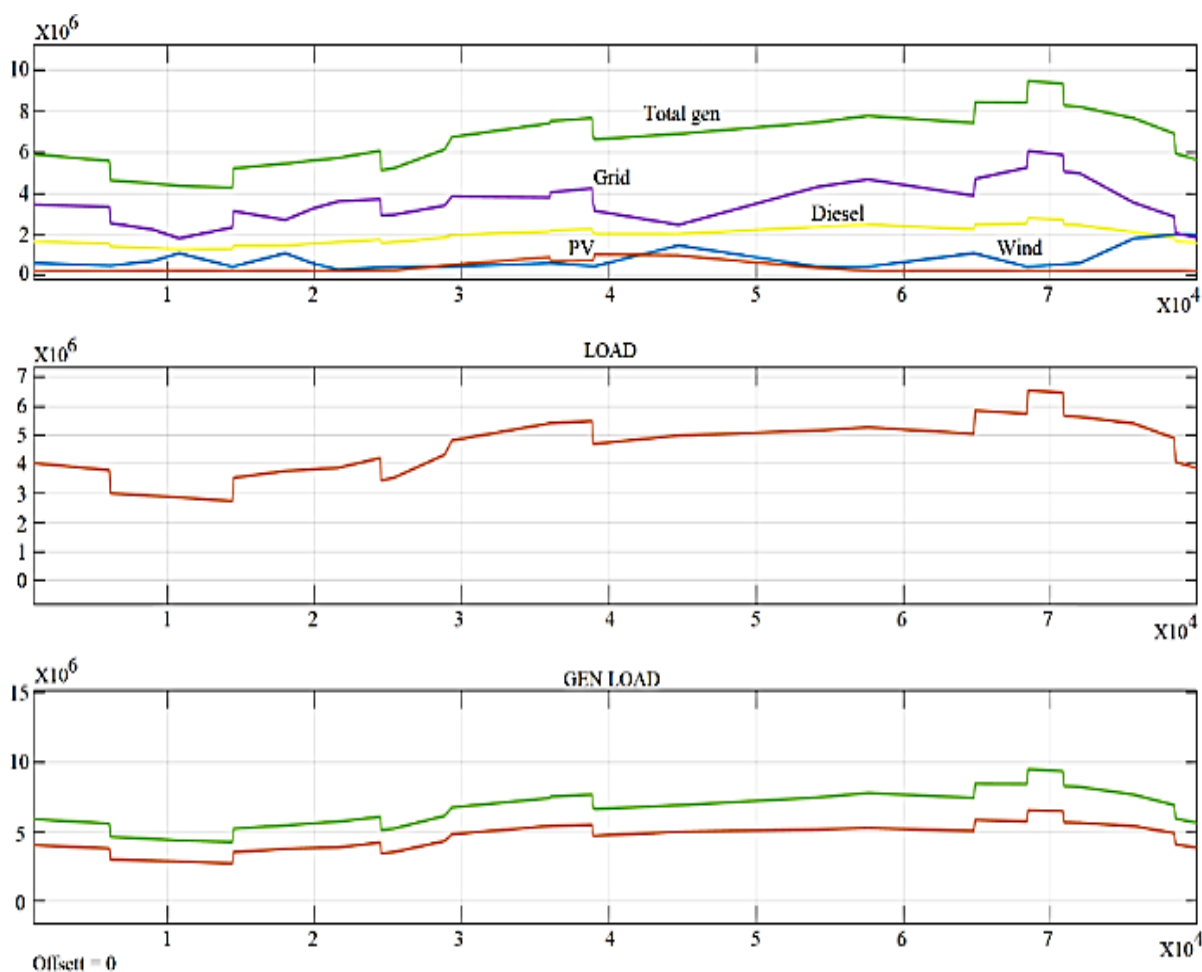

(b)

Figure 12. Results island mode, (a) apparent power simulation result (S) in 24h, (b) system in mode interconnected with the storage system, generators, and load 


\subsection{Interconnected mode}

In this case the governor and exciter of the machine should try to keep the machine at a value no higher than 2 MVA, with which the consumption must be reduced by operating at a lower load point. The machine is intended to regulate the Apparent power of the network. The results are shown in Figure 13 the generator power only exceeds the target value of $2 \mathrm{MVA}$ at the moments of greatest demand. Overpasses are made, in this case the fluctuations of the wind farm. The electrical network (Grid) in violet color has a behavior similar to the load, but the plant is responsible for regulating the stability of the network in times of rapid changes where the grid is insufficient. The grid goes to load the storage system for a greater extent and the diesel plant behaves is much smoother in the interconnection mode with the network [30].

The charge and discharge regulator of the network put some instabilities in the network show as jumps slightly softer than in the interconnected mode. However, the controller is being able to charge faster and demand more energy without affecting the frequency and therefore the load. The controller manages to maintain the frequency in the operation points, with an error of less than $1 \%$, in the cases of the overshoots of the loads or the heaviest changes. The most critical points of operation of the system mode connected are show in the Figure 13, upper box island mode without energy storage, down box in with energy storage.

The frequency regulation keeps the values within less than $0.01 \mathrm{~Hz}$ of error, behaving in an adequate way to the expected. The power remains stable and the frequency regulation system manages to operate the network in island mode and with more complex scenarios such as cogeneration, with or without storage systems. The frequency oscillates in load shedding. The peaks are compensated by the batteries and decrease $(0.009$ to $0.004 \mathrm{Pu})$. Although the oscillations by battery charging processes increase throughout the day.
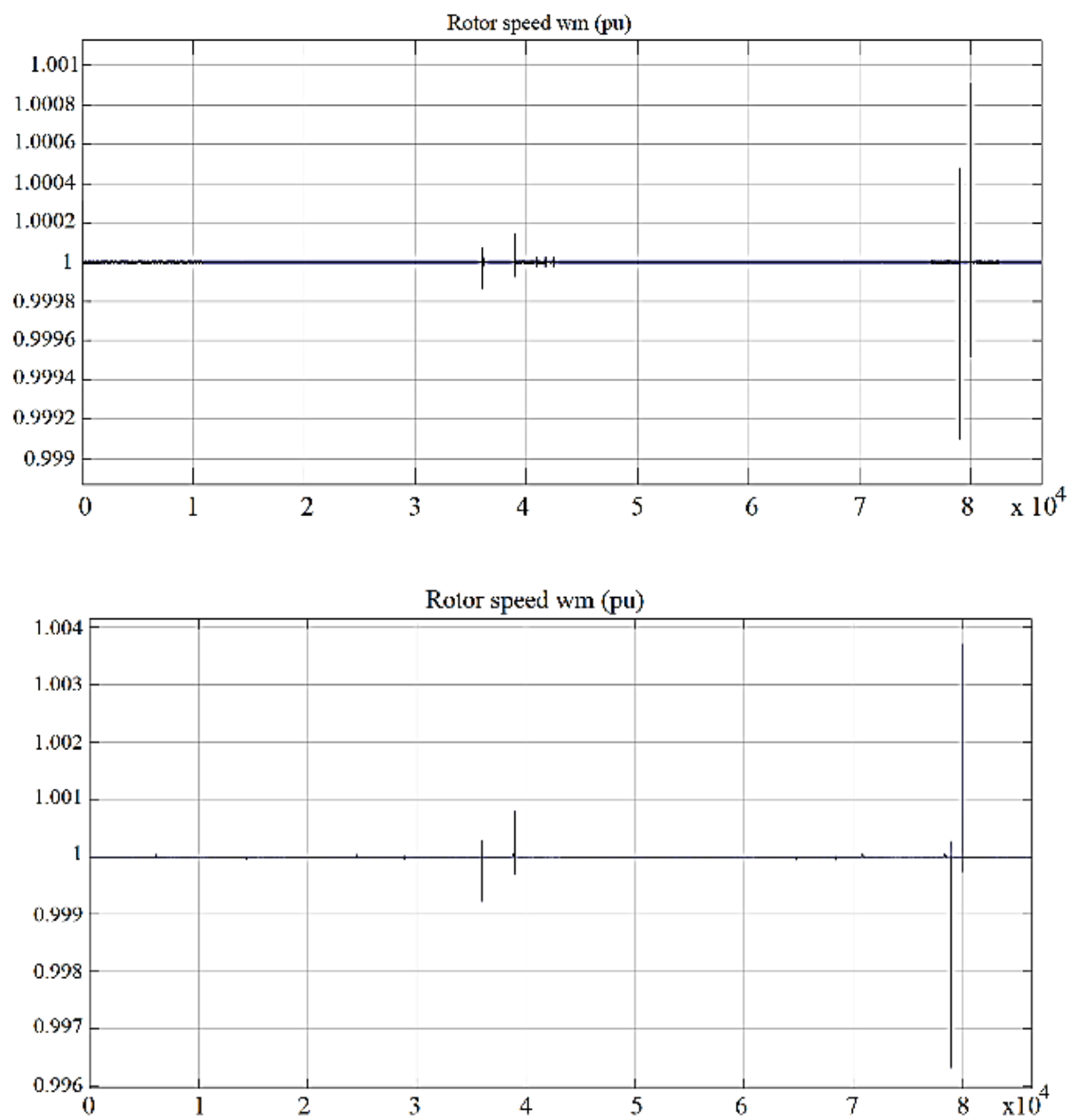

Figure 13. Frequency variation with the system connected island mode without storage (up) vs Frequency variation with the connected island mode with storage (down) 


\section{RESULTS}

The plant manages to compensate the variability of renewable energies the load is variable, and the load flow remains stable There are losses in the system of almost 1MVA, representing the lines, transformation systems and auxiliary power consumption. With power greater than $6 \mathrm{MVA}$ in renewable plants it is not possible to regulate the frequency. If the storage system is tun on the systems show a vary over time, there are batteries in charge and others in discharge. The peaks due to load discharges are larger (20\%) when being fed by the batteries, to keep the frequency stable. The Diesel screen is placed to work at 2MVA, minimizing the frequency $w_{\text {ref }}=0$, to decrease the contribution of the plant. The network sends power to the network (violet).The operation of the plant is much more stable and the electrical network compensates for what the load needs. The sources manage to maintain the load balance.The frequency oscillates in load shedding. The peaks are compensated by the batteries and decrease (0.009 to $0.004 \mathrm{Pu})$. Although the oscillations by battery charging processes increase throughout the day

\section{CONCLUSION}

The simulated model allowed to study the effects of the network in island mode and in interconnected mode, showing the imbalances that can be obtained by integrating renewable energies and storage systems. The proposal with renewable energy sources integrated into the network in alternating current, allowed a modeling of phenomena that occur in the existing network; however, the load flow may not converge if there are many harmonics and power oscillations due to the maneuver of equipment such as switches, so the control cannot be based only on maneuvering equipment and it is suggested on the regulators of voltage and power of each source.

\section{ACKNOWLEDGEMENTS}

The authors are grateful to the Universidad Militar Nueva Granada Military, which through its Vice chancellor for research finances.

\section{REFERENCES}

[1] "Soluciones energéticas para las zonas no interconectadas de colombia," IPSE, 2014.

[2] A. Apostolov, "Integration of Distributed Energy Resources in Smart Grids," 22nd International Conference and Exhibition on Electricity Distribution, pp. 1-5, 2013.

[3] K. Takada, "Smart Grid Related Activity in NEDO: Why Smart grid is needed," 2009.

[4] R. Kempener, et al., "Smart Grids and Renewables - A Guide for Effective Deployment," International Renewable Energy Agency, pp. 1-47, 2013.

[5] X. Fang, et al., "Smart Grid - The New and Improved Power Grid: A Survey," IEEE Communications Surveys and Tutorials, vol. 14, no. 4, pp. 944-980, 2011.

[6] SIEMENS, “Active Power Control in Wind Parks," Siemens Enenrgy, 2011.

[7] R. Perez and T. E. Hoff, "Energy and Capacity Valuation of Photovoltaic Power Generation in New York," Solar Alliance and the New York Solar Energy Industry Association, pp. 1-18, 2008.

[8] IRENA, "Quality Infrastructure for Renewable Energy Technologies - Guidelines for Policy Makers," IRENA, 2015.

[9] A. Marcelo, "Diseño de una planta Solar Fotovoltaica de 50MW," Universidad de la Republica Uruguay, 2016.

[10] IRENA, "REthinking Energy 2017: Accelerating the global energy transformation," IRENA, 2017.

[11] IRENA, "Renewable Energy Statistics 2017,” IRENA, 2017.

[12] S. M. Bitar S. and Fernando C. B., "Estudio de factibilidad para la implementacion de sistemas fotovoltaicos como fuente de energía en el sector industrial de colombia," Colegio de Estudios Superiores de Administracion, Maestria en Administracion de Empresas, Bogota, 2017.

[13] Á. Pinilla, "Manual de Aplicación de la Energía Eólica," Convenio Instituto de Ciencias Nucleares y Energias Alternativas Universidad de Los Andes, 1997.

[14] IEA ETSAP and IRENA, "Renewable Energy Integration in Power Grids. Technology Brief," IRENA, pp. 1-36, 2015.

[15] Kristen Wright, "2011 Utility of the Year: OG\&E," PowerGrid International, 2011. [Online]. Available: http://www.elp.com/articles/print/volume-89/issue-6/features/2011-utility-of-the-year-oge.html.

[16] International Renewable Energy Agency (IRENA), "Smart Grids and Renewables. A Cost-Benefit Analysis Guide for Developing Countries," 2015.

[17] IPSE, "Informe mensual de Telemetria (Puerto Carreño - Vichada)," in Informe Mensual De Telemetría Diciembre 1 - 31 DE 2014, p. 11, 2014.

[18] O. F. A. Sanchez, et al., "Modeling a Microgrid that Integrates Renewable Energies in IEC 61850-7-420 and IEC 61400-25-3," Journal of Engineering Science and Technology Review, vol. 11, no. 4, pp. 174-179, 2018. 
[19] BP, "BP Statistical Review of World Energy 2017," vol. 66, 2017. [Online]. Available: https://www.connaissancedesenergies.org/sites/default/files/pdf-actualites/bp-statistical-review-of-world-energy2017-full-report.pdf.

[20] A. Elgargouri, "Implementation of IEC 61850 in Solar Applications," OSUVA Open Science, 2012.

[21] IEC International Standard, "IEC 61850-7-4 Communication networks and systems in substations - Part 7-4: Basic communication structure for substation and feeder equipment - Compatible logical node classes and data classes," 2010.

[22] IDEAM, "Promedio Horario de la Radiación," IDEAM, 2014.

[23] IDEAM, "Promedio Velocidad del Viento," IDEAM, 2005.

[24] IEEE Standards Association, "IEEE Recommended Practice for Excitation System Models for Power System Stability Studies," IEEE Power and Energy Society, 2006.

[25] M. Elyaqouti, et al., "Modelling and Predicting of the Characteristics of a Photovoltaic Generator on a Horizontal and Tilted Surface," International Journal of Electrical and Computer Engineering (IJECE), vol. 6, no. 6, pp. 2257-2576, 2016.

[26] T. S. Ustun, et al., "Distributed Energy Resources (DER) object modeling with IEC 61850-7-420," AUPEC 2011, pp. 1-6, 2011.

[27] F. A. Chowdhury and D. Le, "Integrated Generation \& Transmission Planning and System Expansion," in 2009 IEEE Power \& Energy Society General Meeting, pp. 1-5, 2009.

[28] G. Xu, et al., "Integrating distributed energy resources in smart grid: Modeling and analysis," IEEE Energytech, Cleveland, OH, pp. 1-5, 2013.

[29] T. Ayodele, et al., "Challenges of Grid Integration of Wind Power on Power System Grid Integrity: A Review," International Journal of Renewable Energy Research, vol. 2, no. 4, pp. 618-626, 2012.

[30] H. Bahri, et al., "Integral Backstepping Control for Maximum Power Point Tracking Factor of a Three Phase Grid Connected Photovoltaic System," International Journal of Electrical and Computer Engineering (IJECE), vol. 7, no. 4, pp. 1671-1680, 2017. 